\title{
An Improved Algorithms of Stroke Generation Considering Multi-level Information Quantity
}

\author{
Liu Yi ${ }^{\text {a,b, },}$, Li Wenjing a,b ${ }^{\text {a }}$ Lin Zhiyong ${ }^{c}$ \\ a School of Resource and Environmental Engineering, Wuhan University of Science and Technology, \\ wtusm_lwj@126.com,906740928@qq.com \\ ${ }^{b}$ Industrial Safety Engineering Technology Research Center of Hubei Province \\ ${ }^{c}$ School of Remote Sensing and Information Engineering, Wuhan University \\ * Corresponding author
}

Keywords: Multi-level information quantity; Stroke; Angle threshold; Douglas-Peucker

\begin{abstract}
:
As a critical component of road network data, stroke has been widely used in analysis and application of road network. However, most conventional stroke generation strategies are based solely on a regular angle threshold, ignoring the structural characteristics of the road network. In fact, map information comes from the diversity and difference of elements and their distribution characteristics. Different road network structures should also correspond to different angle thresholds, rather than a fixed value. Therefore, quantitatively describing the structure characteristics of road network, has an important impact on the determination of the threshold in stroke generation strategies. In this paper, we propose an algorithm of stroke generation based on spatial feature information, which achieves the selection of the optimal threshold under different road network structures.
\end{abstract}

The information quantity of map is generated by the diversity and difference of elements and their distribution characteristics. In other words, the greater the diversity and difference between strokes, the greater the corresponding information quantity generated. The traditional Shannon information entropy theory is based on the probability of the occurrence of map symbols. If different symbols have the same probability of occurrence, their information will be the same, which can not reflect the structural characteristics of the road network. For this reason, this paper adopts a model based on spatial feature quantities. By taking the spatial information characteristics of strokes as the measurement indicators, the model takes into account the differences and spatial distribution characteristics of stroke, and calculates the information quantity of different road network structures. The expression is as follows:

$$
I=\sum_{i=1}^{n} \log _{2}\left(V_{i}+1\right)
$$

where $V_{i}$ represent a measurement indicator for expressing the geometric and topological characteristics of stroke, and $I$ is information quantity of road network.

According to the spatial characteristics of different levels, the spatial information of road network correspondingly consists of geometric information at element level and topological information at neighborhood level. By selecting the corresponding indicators of road elements and neighborhood levels, quantitatively calculates the road network information under different angle thresholds; Secondly, the Douglas-Peucker algorithm is used to determine the optimal threshold for stroke-generate under different road network structures. Finally, the global efficiency commonly used in complex network theory, describes the degree of smoothness for information dissemination in the network and can be used evaluate the result of stroke generation. Figure 1 shows a flow chart for the improved stroke algorithm. 


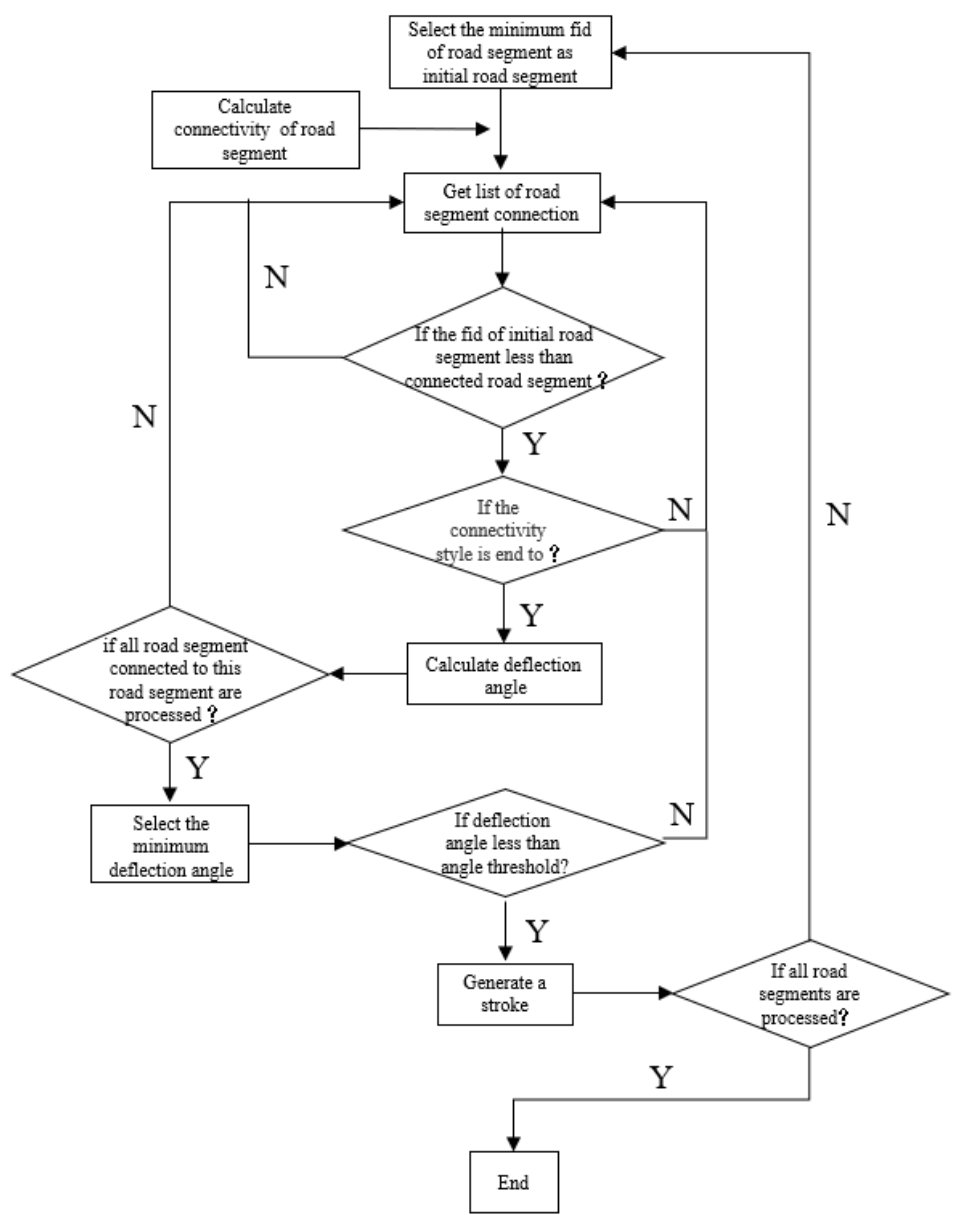

Figure 1. A flow chart for the improved stroke algorithm 